\title{
Diode laser contact transscleral retinal photocoagulation: a clinical study
}

Moorfields Eye Hospital, London D A McHugh S Schwartz J G Dowler M Ulbig R K Blach P A Hamilton

Correspondence to: Mr Dominic McHugh, King's College Hospital, Denmark Hill, London SE5 9RS.

Accepted for publication 8 August 1995

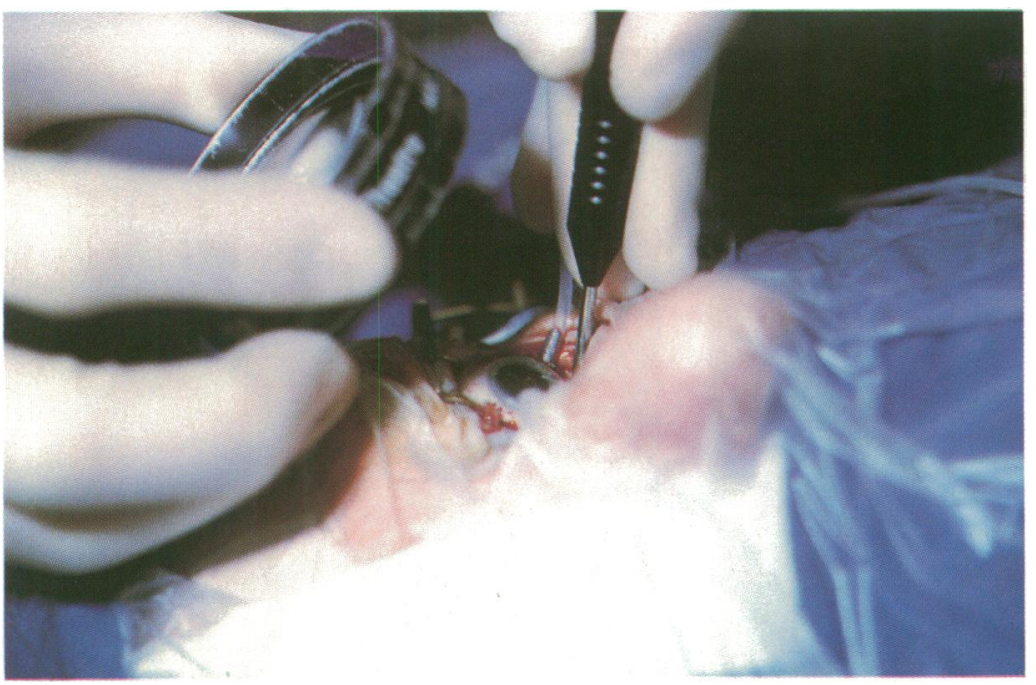

Figure 1 Transscleral delivery of diode laser radiation using a specialised probe. The target area and the transilluminated aiming beam are visualised with a $20 \mathrm{D}$ lens. treatment of retinal vascular conditions and glaucoma. ${ }^{1-3}$ The portability and reliability of diode lasers confer ergonomic advantages over ion lasers; and the infrared emission wavelength has the biophysical advantages of good transmission through haemorrhagic media opacities and nuclear sclerotic cataract, together with negligible absorption within macular xanthophyll. ${ }^{45}$

The initial delivery systems employed transpupillary irradiation of the target zone (in conjunction with, for example, a slit-lamp microscope). More recently, however, the high scleral transmission of near infrared radiation has prompted interest in the employment of diode lasers for transscleral therapy. Pilot studies have reported the effectiveness of diode laser cyclophotocoagulation in the treatment of refractory glaucoma and histological work and a pilot clinical study have indicated the potential of transscleral retinal therapy ('retinopexy'). ${ }^{6-8}$ Stated advantages include the ability to bypass media opacities, which would otherwise attenuate the treatment beam, and also less marked inflammatory side effects in comparison with transscleral cryotherapy.

The aims of the current study were to examine the potential range of applications of transscleral diode laser retinopexy in the treatment of surgical retinal conditions, to optimise treatment variables, and to assess whether this novel modality had significant advantages over conventional techniques.

\section{Materials and methods}

LASER

All treatments were performed using a semiconductor diode laser, with an emission wavelength of $810 \mathrm{~nm}$ (Iris Medical Instruments Inc, Mountain View, CA, USA). The maximum available power was $2 \mathrm{~W}$ and the exposure duration could be incrementally increased up to a maximum of 9 seconds. Laser delivery was accomplished via a transscleral probe, which was applied to the external scleral surface (Fig 1).

The probe tip incorporated a $2 \times 2 \mathrm{~mm}$ glass prism, which had a $1.5 \mathrm{~mm}$ diameter output face, to deliver laser energy perpendicular to the device handle and shaft. The face of this prism protruded $0.8 \mathrm{~mm}$ above the shaft shank, permitting indentation at the treatment site. The target area was visualised with an indirect ophthalmoscope while a transilluminating aiming beam from a red emitting diode laser facilitated precise placement of the probe over the target area. 


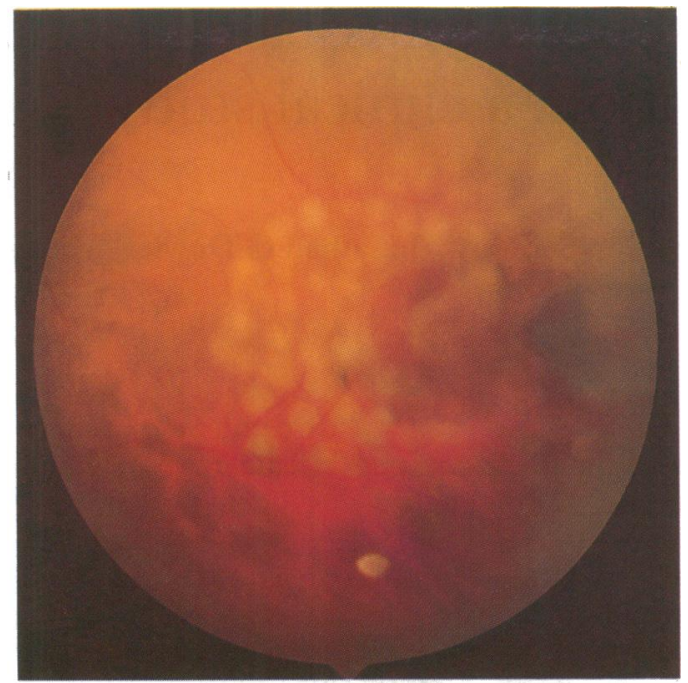

Figure 2 Acute burns produced by transscleral diode laser treatment of a peripheral retinal break. Note the grey-white appearance of the lesions, which is typical of irradiation with a wavelength of $810 \mathrm{~nm}$.

Optics inside the probe produced an aerial image of the laser fibre optic, $0.9-1.0 \mathrm{~mm}$ in diameter at the 'ideal' treatment plane located $0.5 \mathrm{~mm}$ from the output face of the prism. Residual beam divergence near the prism face was only approximately $80 \mathrm{mrad}$ full angle. This means that within $1 \mathrm{~mm}$ from the output face of the prism, the laser illuminated spot did not change by more than $0.1 \mathrm{~mm}$.

A foot switch activated the laser and in all cases the desired visible end point was the greyish-white appearance typical of a threshold diode laser lesion. The strong forward scattering characteristics of sclera imply that the diameter of the treatment beam was not significantly increased during its passage through the sclera.

\section{PATIENTS}

A total of 36 eyes in 36 patients underwent diode laser retinopexy. The mean age was 47 (range 18-45) years and the mean period of review was 8 (range 4-12) months. There were a number of indications for transscleral diode laser retinopexy:

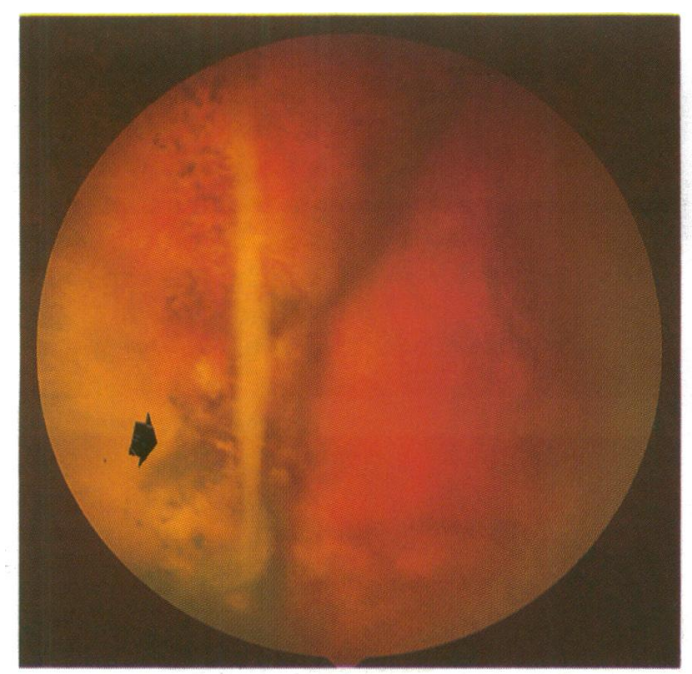

Fig $3 A$ (a) Retinal tears of up to 2 clock hours in extent, which were associated with retinal detachment (30 eyes). After drainage of subretinal fluid (where appropriate), each break was surrounded by two rows of laser burns. This was then followed by application of a scleral explant and, if necessary, air or gas injection.

(b) Irradiation of the site of drainage of subretinal fluid. In those cases of retinal detachment which required drainage (28 eyes), the technique of external suture needle drainage was applied. ${ }^{9}$ With the aim of inducing choroidal vascular closure and thus reducing the risk of drainage related haemorrhage, a pattern of 16 exposures was applied to the location selected for drainage.

(c) Giant retinal tears (six eyes). Tears of 3 clock hours or more in extent were treated with vitrectomy and silicone oil exchange. A double row of diode laser burns was administered to the posterior edge of the break and the 'horns' of the tear.

During treatments for these conditions, the initial power setting was $500 \mathrm{~mW}$ and the target area irradiated until a reaction was observed. The power was increased in 250 $\mathrm{mW}$ increments if a lesion was not observed after an exposure of up to 2-3 seconds. The power was decreased following an intense blanching reaction, an audible 'popcorn' effect, or a haemorrhagic lesion. All procedures were performed subconjunctivally under peribulbar or general anaesthesia. Careful note was made of any peroperative or subsequent side effects of treatment. Patient review was at 2 weeks, 6 weeks, and 12 weeks after therapy, then at 3 monthly intervals.

\section{Results}

The irradiances required for threshold lesions ranged between $95.4 \mathrm{~W} / \mathrm{cm}^{2}$ and $191 \mathrm{~W} / \mathrm{cm}^{2}$ (mean 133 (SD $27 \cdot 20) \mathrm{W} / \mathrm{cm}^{2}$ ), with treatment variables $0 \cdot 75-1 \cdot 5 \mathrm{~W}$ power and $1-4$ second pulse duration. Fundus pigmentation influenced the treatment variables selected, the two non-white patients (who were Asian-Indian) requiring lower irradiances than

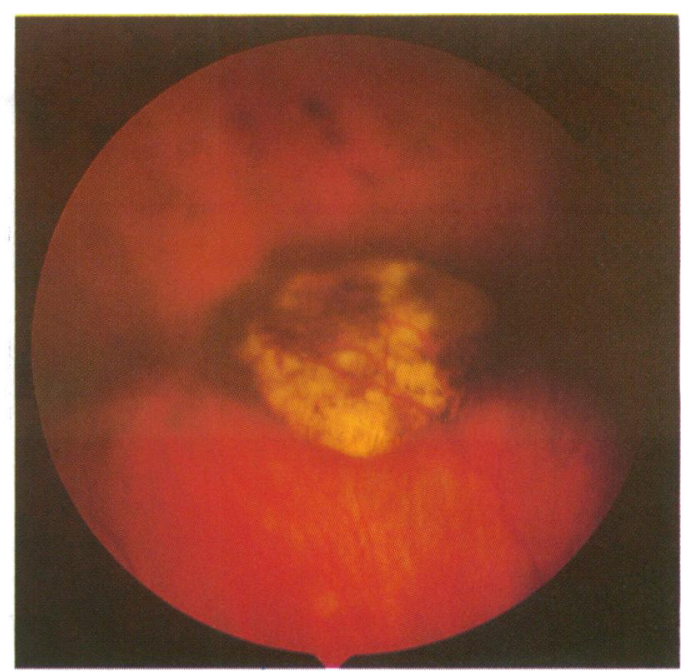

Fig $3 B$
Figure 3 (A) Six months break during retinal become pigmented scar (arrowed). (B) In the same eye, prophylactic cryotherapy had been 
the white patients $\left(95.4 \mathrm{~W} / \mathrm{cm}^{2}\right.$; power $0.75 \mathrm{~W}$ and pulse duration 1 second). Higher powers were required in the presence of subretinal fluid or haemorrhage. Other relevant factors included the need to place the probe precisely perpendicular to the scleral surface (which was indicated by how sharply defined the aiming beam appeared), and whether treatment was being applied through an extraocular muscle. Retinal blanching could usually be seen even in cases where the fundal view was obscured by subretinal fluid, cataract, or blood. It was noticeable that even extensive treatments did not induce chemosis, or lid oedema, as is commonly observed after cryotherapy.

In those eyes with retinal tears and retinal detachments, effective chorioretinal lesions were produced with transscleral therapy (Fig 2). Retinal reattachment was initially achieved in all the eyes, although the subsequent formation of new breaks required a second (nondiode) vitrectomy procedure in two eyes. In neither case was this thought to be a diode laser related complication. Punctate haemorrhages were observed at the centre of several lesions in three eyes, but reduction in power eliminated this effect in subsequent exposures.

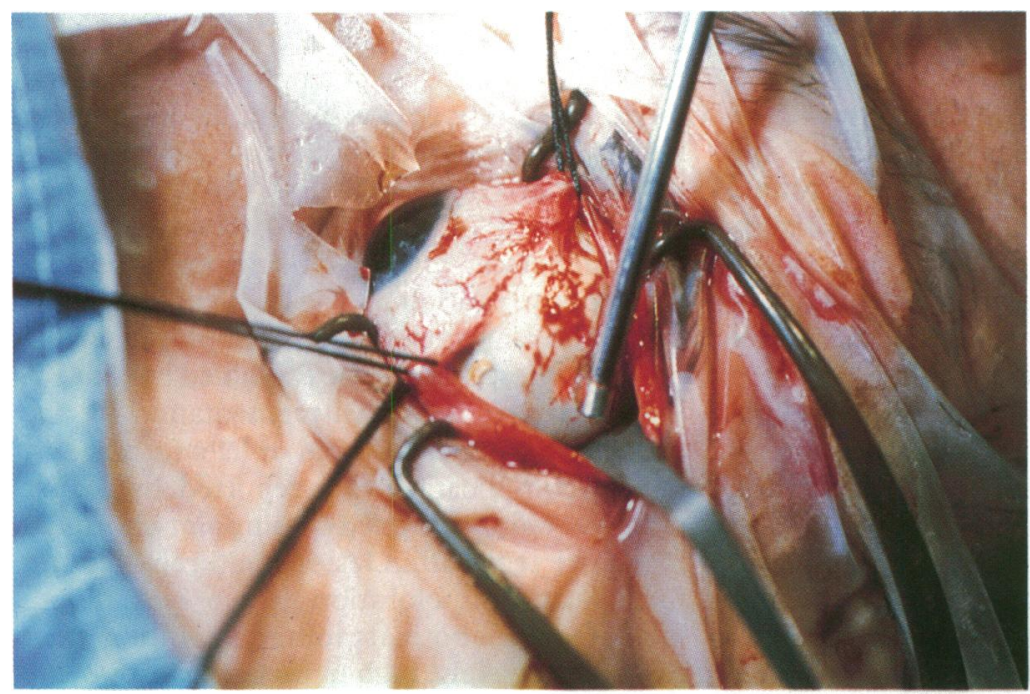

Fig $4 A$

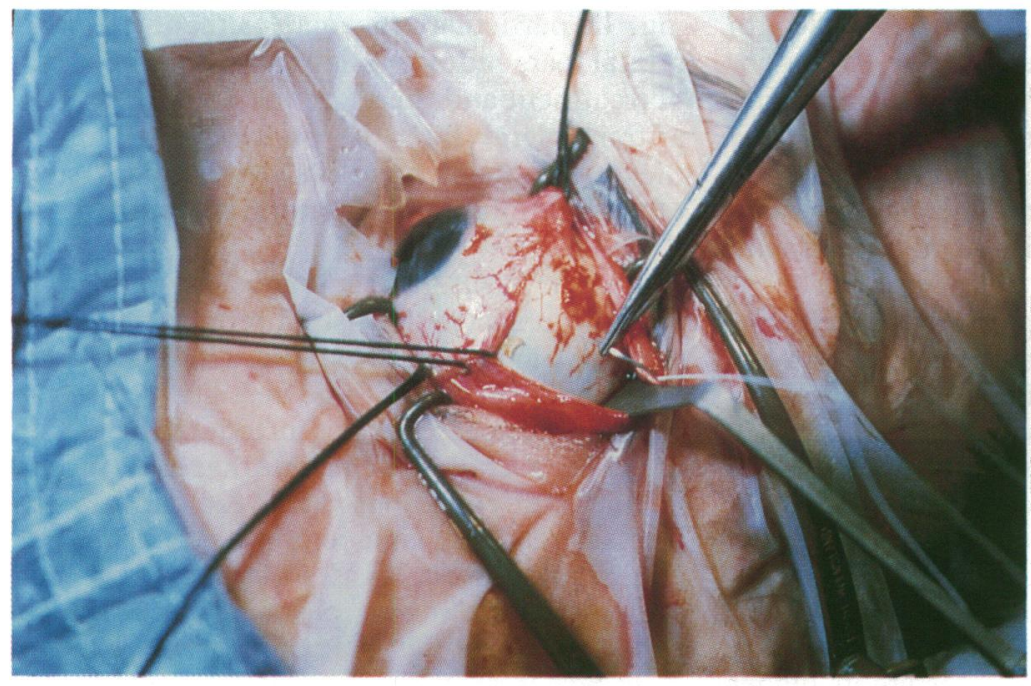

Fig $4 B$
Retinal burns 12 weeks after treatment appeared as pigmented scars. One eye had undergone prophylactic cryotherapy several years previously. In contrast with diode laser scars there was a greater amount of chorioretinal atrophy associated with the cryopexy scar (Fig 3). Retinopexy before transscleral suture needle drainage of subretinal fluid was followed by drainage induced haemorrhage of 2-3 disc diameters in area in two of 28 eyes. No drainage related complications were observed in the remaining eyes (Fig 4).

Retinal reattachment was achieved in all of the eyes treated for giant retinal tears. One striking feature was the mild degree of postoperative inflammation. The relatively extensive photocoagulation required induced relatively little intravitreal pigment dispersion or uveitis, in comparison with that commonly seen with extensive retinal cryotherapy. In one eye an excessively high power induced several punctate choroidal haemorrhages, but these had resolved by 2 weeks postoperatively.

\section{Discussion}

The results of this study suggest the potential of transscleral diode laser therapy in the treatment of surgical conditions of the retina and that there may be significant advantages over techniques currently employed.

The principal aims of retinal reattachment surgery are to appose the neurosensory retina to the underlying retinal pigment epithelium and create a chorioretinal adhesion around all retinal breaks. The three principal methods available to produce a chorioretinal scar are diathermy, cryotherapy, and photocoagulation. The ideal requirement of a transscleral delivery system is the ability to induce a focal tissue effect that is limited to the outer retina and inner choroid, with minimal damage to the inner retina, outer choroid, or sclera.

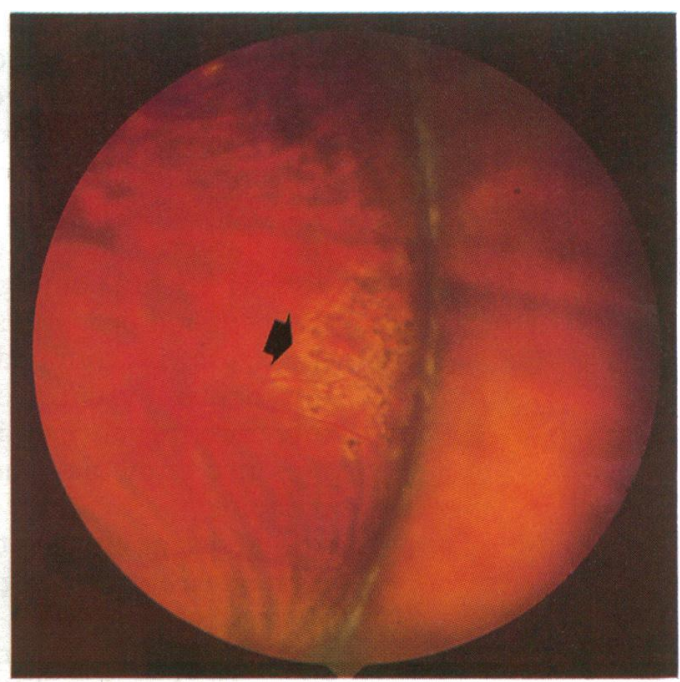

Fig $4 C$

Figure 4 (A) Transscleral diode laser retinopexy to the area selected for drainage of subretinal fluid. The aim was to decrease the risk of drainage related choroidal haemorrhage. (B) Drainage of subretinal fluid using a suture needle at the site of earlier photocoagulation. (C) Diode laser scars in the site of drainage of subretinal fluid (arrowed). 
Table 1 Transmission and absorption characteristics of visible and infrared wavelengths within ocular tissues

\begin{tabular}{lllll}
\hline & $\begin{array}{l}\text { Argon green } \\
(514 \cdot 5 \mathrm{~nm})\end{array}$ & $\begin{array}{l}\text { Krypton red } \\
(647 \mathrm{~nm})\end{array}$ & $\begin{array}{l}\text { Diode } \\
(810 \mathrm{~nm})\end{array}$ & $\begin{array}{l}\text { Nd:YAG } \\
(1064 \mathrm{~nm})\end{array}$ \\
\hline $\begin{array}{l}\text { Scleral transmission }(\%)^{13} 14 \\
\quad \text { Non-contact }\end{array}$ & 12 & 18 & 35 & 53 \\
$\begin{array}{l}\text { Indentation } \\
\text { Absorption (\%) } \\
\quad \text { Melanin }\end{array}$ & 33 & 43 & 71 & 77 \\
\hline
\end{tabular}

${ }^{\star}$ Choroid and retinal pigment epithelium. ${ }^{15}$

Before the introduction of cryotherapy, diathermy was the preferred method of inducing a chorioretinal adhesion in the treatment of retinal breaks. ${ }^{10}$ The physical principles of diathermy involve the generation of radiofrequency energy that is converted to heat as it propagates through biological tissue. It is, therefore, relatively non-selective in its site of action and, in addition to producing a chorioretinal lesion, also causes scleral destruction and weakening and extensive choroidal destruction, with damage to vortex veins or the posterior ciliary arteries or nerves.

Cryotherapy, which produces an inflammatory reaction and thus chorioretinal adhesion by tissue freezing, has supplanted diathermy in the transscleral treatment of retinal breaks. It produces little scleral damage ${ }^{11}$ but a similar strength of chorioretinal adhesion. Cryotherapy, however, does have a number of potentially serious side effects, which include a marked breakdown in the integrity of the blood-retinal barrier and dispersion of retinal pigment epithelial cells into the vitreous cavity. The combination of serum derived factors and pigment epithelial cells may increase the risk of proliferative vitreoretinopathy (PVR) and of failure of retinal detachment surgery. ${ }^{12}$

The essential mechanism of action of retinal laser photocoagulation is the absorption of radiant energy by a chromophore, melanin, within the retinal pigment epithelium and choroid and its conversion to heat, with subsequent tissue coagulation. The initial coagulation, followed by secondary gliosis, accounts for the adhesive effect of lesions. The majority of lasers that are currently employed for this purpose emit radiation in the visible part of the electromagnetic spectrum - for example, the argon laser (emission wavelength 488-514.5 nm, blue-green). They have poor transmission properties through the sclera and their use is confined to transpupillary or intraocular systems of delivery. They are, therefore, of more limited effectiveness in the presence of media opacities or very peripheral pathology.

The recent introduction of lasers which emit in the near infrared region of the spectrum has allowed consideration of alternative delivery systems. The radiant emissions of the Nd:YAG laser $(1064 \mathrm{~nm})$ and the semiconductor diode laser $(810 \mathrm{~nm})$ have a combination of high scleral transmission and significant absorption within melanin. In contrast, several studies have demonstrated that argon laser irradiation is poorly transmitted through the sclera, even with indentation. Therefore, despite its higher absorption within melanin than the infrared wavelengths, it has not been considered appropriate for transscleral applications (Table 1, refs 13-15). In relation to the diode laser, histological studies of transscleral irradiation demonstrated its ability to produce both ciliary body and chorioretinal lesions, but with no evidence of thermal damage to the sclera. ${ }^{7} 16$ High transmission through silicone explants may also be of advantage in the retreatment of eyes with retinal breaks (Nanda et al; poster presentation, ARVO, 1993).

There is also evidence that diode laser retinopexy induces less pigment epithelial cell dispersion than cryotherapy, with important implications for the relative risks of induction of PVR following each form of therapy (Nissen et al; poster presentation, ARVO, 1993). Another study specifically compared bloodretinal barrier breakdown in rabbits induced by transscleral diode laser irradiation and retinal cryotherapy. Magnetic resonance imaging with gadolinium-DPTA enhancement of equal areas of treated retina demonstrated a twofold increase in signal enhancement in eyes treated with cryotherapy, compared with diode laser treated eyes. The difference in signal enhancement was correlated directly with change in permeability of the blood-retinal barrier (Arrindell et al; poster presentation, ARVO, 1992).

In a pilot study, Haller and colleagues reported their success in employing transscleral diode laser retinopexy in retinal detachment surgery. ${ }^{8}$ Lesions were produced using energies which ranged between 234 and $1492 \mathrm{~mJ}$ (white patients) and 74 and $251 \mathrm{~mJ}$ (black patients), the differential energies being explained by pigmentary variation. Although generally higher radiant energies were employed in the present series, this may be explained by individual differences in fundal pigmentation, or the presence of retinal oedema, or haemorrhage.

The complication rate reported by Haller was low, being confined to pinpoint choroidal haemorrhages within lesions in three eyes and focal scleral discoloration in two eyes. These incidents were both attributed to employment of an excessively higher power. Despite the limited sample sizes in that and the current study, the low incidence of PVR following transscleral diode laser therapy is encouraging. In particular, the management of giant retinal tears requires relatively extensive areas of treatment and therefore the employment of a transscleral laser in preference to cryotherapy may minimise intravitreal dispersion of the cellular and humoral precursors of PVR.

The use of suture needle drainage of subretinal fluid obviates the risk of retinal incarceration that may follow scleral dissection and choroidal perforation. The inability to directly cauterise the choroid does introduce the risk of inducing a choroidal haemorrhage. A recent animal study demonstrated the efficacy of transscleral diode laser retinopexy in achieving choroidal vascular stasis sufficient to prevent haemorrhage following passage of a 20 gauge needle through the irradiated areas (Kaplan et al; poster presentation, ARVO, 1993). The low incidence of haemorrhagic complications 
of drainage in the present study tends to confirm these findings. The procedure is easy to perform, the only potential difficulty being in visualisation of choroidal lesions in the presence of deep or turbid subretinal fluid.

The successful deployment of the transscleral probe requires awareness of its differences from retinal cryotherapy. It is important to maintain the probe tip perpendicular to the sclera while indenting in order to maximise scleral transmission, and the aiming beam must be in sharp focus. In contrast with cryotherapy, laser photocoagulation is sensitive to variations in fundal pigmentation and the smaller areas of laser lesions does increase the treatment time compared with cryotherapy. Localisation of small retinal breaks with cryotherapy is enhanced by the contrast of retinal whitening seen around the hole, an effect more difficult to achieve with a laser. However, although the immediate cryotherapy reaction tends to fade, a laser lesion remains clearly visible, and thus the risks of overtreatment are reduced.

This study has given an indication of the potential role of transscleral diode laser retinopexy in the treatment of surgical retinal conditions. The low complication rate and the early indications of clinical efficacy provide justification for the establishment of formal trials of its use in individual conditions.

Each author states that he has no proprietary interest in the development or marketing of this or a competing instrument.

1 McHugh JDA, Marshall J, ffytche TJ, Hamilton PAM, Raven A, Keeler CR. Initial clinical experience using a diode laser in the treatment of retinal vascular disease. Eye 1989; 3: 516-27.

2 Balles MW, Puliafito CA, D'Amico DJ, Jacobson JJ, Birngruber P. Semiconductor diode laser photocoagulation in retinal vascular disease. Ophthalmology 1990; 97: 1553-61.

3 McHugh D, Marshall J, ffytche TJ, Hamilton PAM, Raven A. Diode laser trabeculoplasty (DLT) for primary open angle glaucoma and ocular hypertension. $\mathrm{Br} f \mathrm{Ophthalmol}$ 1990; 74: 743-7.

4 Horeckler BL. The absorption spectrum of haemoglobin and its derivatives in the visible and near infrared regions. and its derivatives in the visible

5 Nussbaum JJ, Pruett RC, Delori RC. Macular yellow pigment: the first 200 years. Retina 1981; 1: 296-310.

6 Gaasterland DE, Pollack IP. Initial experience with a new method of laser transscleral cyclophotocoagulation for ciliary ablation in severe glaucoma. Trans Am Ophthalmo Soc 1992; 90: 225-43.

7 Jennings T, Fuller T, Vukich J, Lam T, Joondeph BC, Ticho B, et al. Transscleral contact retinal photocoagulation with an $810 \mathrm{~nm}$ semiconductor diode laser. Ophthalmic Surg 1990; 21: 492-6.

8 Haller J, Lim J, Goldberg M. Pilot trial of transscleral diode laser retinopexy in retinal detachment surgery. Arch laser retinopexy in retinal
Ophthalmol 1993; 111: $952-6$.

9 Raymond GL, Lavin MJ, Dodd CL, McLeod D. Suture needle drainage of subretinal fluid. Br $\mathcal{F}$ Ophthalmol 1993; 77: 428-9.

10 Schepens CL. Retinal detachment and allied diseases. Philadelphia: WB Saunders Co, 1983: 289-99.

11 Steinmetz CG, III. Reaction of the sclera to cryosurgery. Int Ophthalmol Clin 1967; 7: 395.

12 Glaser BM, Vidaurri-Leal J, Michels RG, Campochiaro PA. Cryotherapy during surgery for giant retinal tears and
intravitreal dispersion of viable RPE cells. Ophthalmology intravitreal dispersion

13 Rol P, Niederer P, Durr U, Henchoz PD, Fankhauser F. Experimental investigations on the light scattering properties of the human sclera. Lasers Light Ophthalmol 1990; 3: 201-12.

14 Vogel A, Dlugos C, Nuffer R, Birngruber R. Optical properties of human sclera and their consequences for transscleral laser applications. Lasers Surg Med 1991; 11: $331-40$.

15 Geeraets WJ, Berry BS. Ocular spectral characteristics as related to hazards from lasers and other light sources. $\mathrm{Am}$ related to hazards from lasers and

16 Hennis HL, Assia E, Stewart WC, Legler UFC, Apple DJ. Transscleral cyclophotocoagulation using a semiconductor diode laser in cadaver eyes. Ophthalmology 1991; 21 274-8. 